\title{
Examining the Safety and Efficacy of Low-Level Laser Therapy for Male and Female Pattern Hair Loss: A Review of the Literature
}

\author{
Andjela Egger $^{\mathrm{a}}$ Sydney R. Resnik ${ }^{\mathrm{a}, \mathrm{b}}$ Divya Aickara ${ }^{\mathrm{a}}$ Eric Maranda $^{\mathrm{a}}$ \\ Michael Kaiser $^{a}$ Tongyu C. Wikramanayake ${ }^{a}$ Joaquin J. Jimenez ${ }^{a}$ \\ aDr. Phillip Frost Department of Dermatology and Cutaneous Surgery, University of Miami Miller School of Medicine,

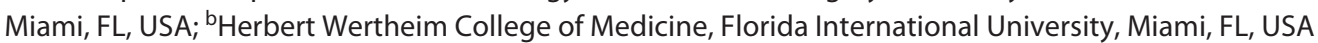

\section{Keywords}

Low-level laser therapy · Androgenetic alopecia · Female pattern hair loss · Alopecia

\begin{abstract}
Purpose: Pattern hair loss is the most common type of alopecia. Standard of care involves long-term use of topical medications with limited effectiveness. Low-level laser therapy (LLLT) has become a popular alternative treatment. Here, we examine published clinical trials to establish whether the breadth of evidence supports LLLT for pattern hair loss. Methods: A literature search was conducted within the PubMed, Embase, Scopus, and Cochrane Trials databases to identify original articles evaluating hair regrowth following LLLT. Articles were selected based on use of $600-1,100 \mathrm{~nm}$ wavelengths, treatment time $\geq 16$ weeks, and objective evaluation for hair regrowth. Results: Ten randomized controlled trials were included, of which 8 compared LLLT to sham device and 1 to no treatment. The study populations varied, with 3 studies evaluating only women. All sham-device controlled studies demonstrated statistically significant increase in hair diameter or density $(p<0.01)$ following LLLT. Discussion: Based on our review of the literature, LLLT appears to be effective for treating pattern hair loss in both men and
\end{abstract}

women. These laser devices have good safety profiles, with only minor adverse effects reported. However, physicians should be cautious when drawing conclusions as some studies included have a relationship with industry.

(c) 2020 S. Karger AG, Basel

\section{Introduction}

Pattern hair loss, also known as androgenetic alopecia (AGA), or male pattern hair loss (MPHL) in males and female pattern hair loss (FPHL) in females, is the most common type of alopecia [1]. Prevalence increases with age, and a study found that $57 \%$ of women and $73.5 \%$ of men over the age of 80 suffered from pattern hair loss [2]. Because hair is an important aspect of human appearance, which is commonly used for identification and is one factor of physical appeal, hair loss can lead to diminished quality of life and flawed social worth [3]. Individuals suffering from hair loss find the experience stressful and report negative body image, low self-esteem, and loss

A.E. and S.R.R. have had substantial and equal contributions to the manuscript.

$\begin{aligned} & \text { karger@karger.com } \\ & \text { www.karger.com/sad }\end{aligned}$
Karger ${ }^{\prime /}$


Table 1. Jadad criterion scoring

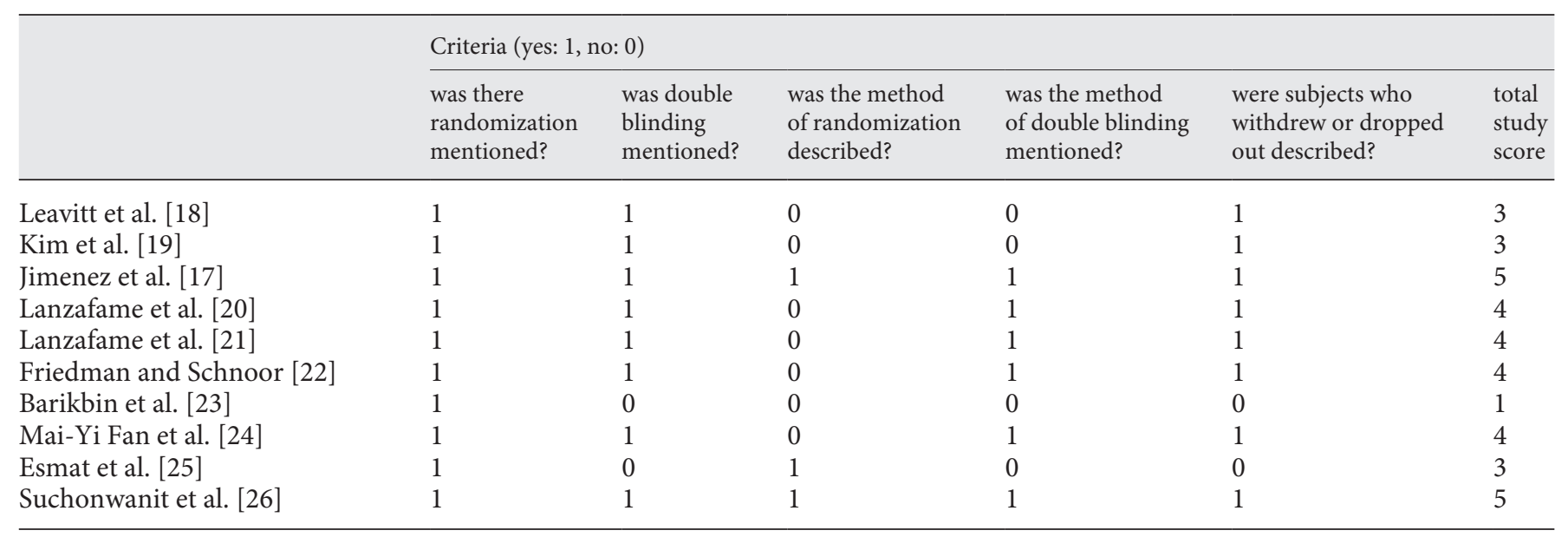

of self-confidence $[4,5]$. This tends to be magnified in women, who were shown to find hair loss more distressing than men and do not adapt as well as men $[4,6,7]$.

MPHL, also known as AGA, is characterized by a progressive decline in hair fiber production by scalp hair follicles and their subsequent miniaturization. MPHL/AGA is due to a combination of genetic predisposition and the effect of androgens. Although testosterone is necessary for the development of male balding, its more potent metabolite, dihydrotestosterone, is responsible for influencing follicular regression [8]. While the role of androgens in MPHL is well illustrated, its role in FPHL has been questioned [9].

Established medical management for pattern hair loss to date consists of $5 \alpha$-reductase inhibitors (finasteride and dutasteride) and topical minoxidil, which require frequent and indefinite use and have limited effectiveness [10]. Minoxidil acts as a potassium channel opener on the smooth muscles of the peripheral arteries. It is postulated that this potassium channel activity is required for progression to the G1 stage of the cell cycle, cellular proliferation, and ultimately in this case hair growth $[11,12]$. Surgical options are restricted to patients due to high cost and supply of donor hair follicles [10, 13].

Due to the need for more successful therapies, lowlevel laser therapy (LLLT) has emerged as a novel therapy to treat pattern hair loss. LLLT has become widely popular due to commercially available devices that can be used at home, are of low cost, are easy to navigate, and have a great safety profile. To date, there are 29 FDA cleared devices for the treatment of pattern hair loss in males, females, or both [14]. LLLT has biostimulatory effects on tissues and is presumed to prolong anagen (growth phase), stimulate anagen reentry from telogen (resting phase), and inhibit early transition to catagen (regression phase) $[9,15,16]$. Additionally, they stimulate the production of terminal hair from follicles that have been producing pseudo-vellus hair [17]. However, there is still considerable skepticism from the dermatology community about its use to promote hair growth due to lack of literature reviews looking at overall benefits of LLLTs compared to other modalities, limited understanding of mechanisms, and inadequately defined treatment parameters. For this reason, the purpose of this review is to examine published controlled clinical trials, which include comparison of LLLT devices to sham device or no treatment for their safety and effectiveness for the treatment of pattern hair loss in both males and females.

\section{Methods and Materials}

\section{Literature Search}

A broad literature search was conducted through PubMed, Embase, Scopus, and Cochrane Trials up to January 2020 to identify original articles that evaluate hair regrowth upon LLLT. The databases were searched using different combinations of the following keywords: alopecia, pattern hair loss, androgenetic alopecia, hair loss, hair regrowth, low level laser therapy, low level light therapy, photobiomodulation, or low energy laser irradiation.

\section{Inclusion and Exclusion Criteria}

All search hits from all 4 databases were screened and examined for relevant abstracts and titles. Full text of relevant studies was reviewed to determine appropriateness according to established inclusion criteria. Case reports and case series were excluded. Articles were recognized as appropriate when they (a) included hu- 


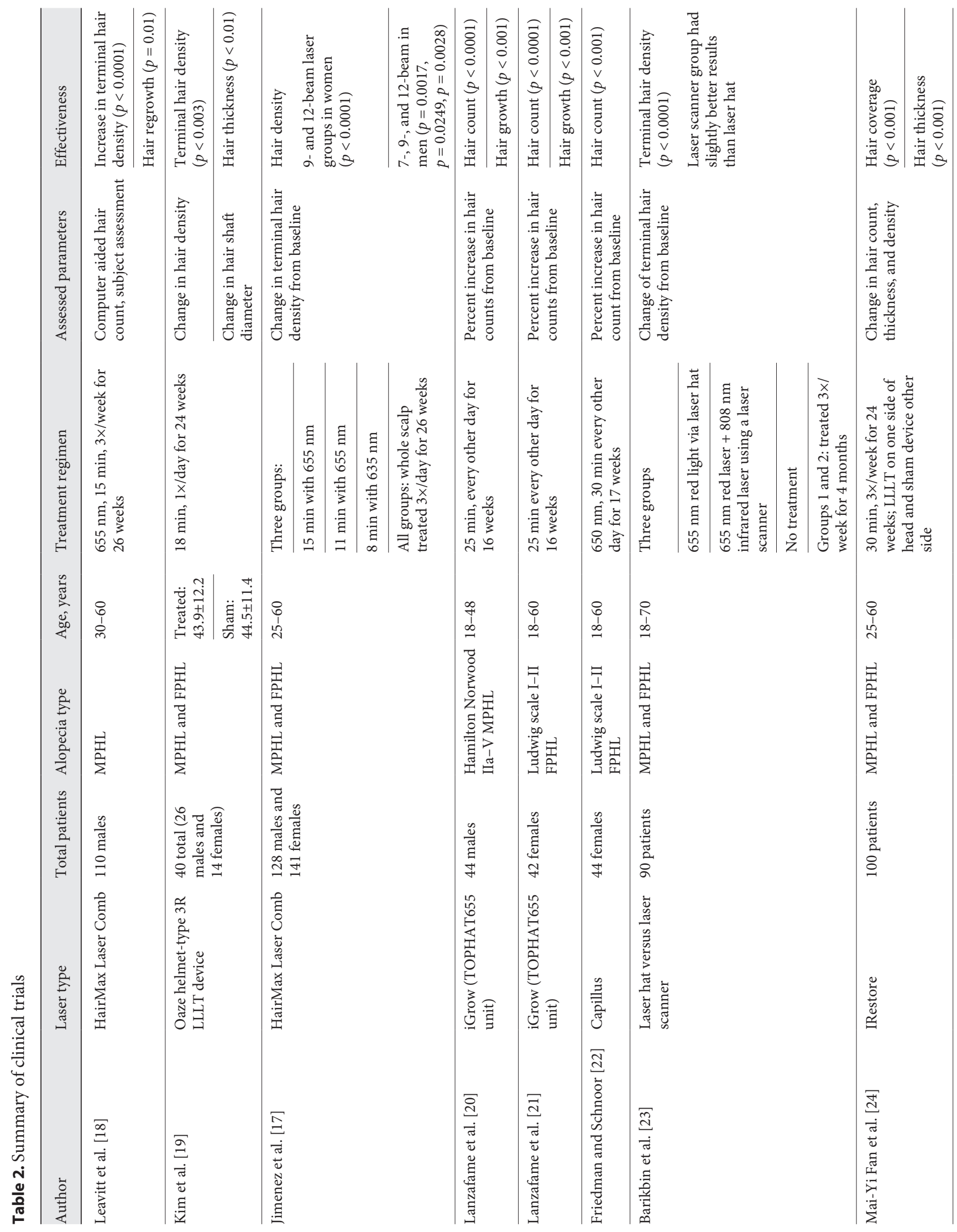




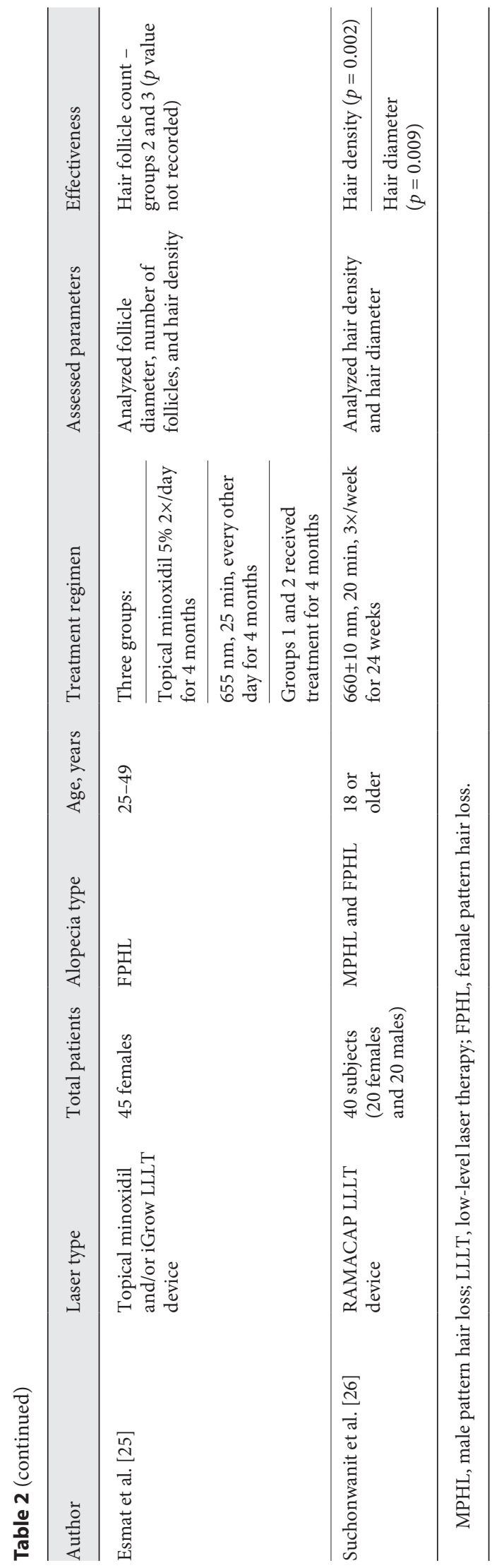

man subjects, (b) used a wavelength between 600 and 1,100 nm, (c) had a control group, (d) used an objective measure for hair regrowth, and (e) involved a 16-week or greater clinical trial.

Quality of Evidence

The quality of each of the papers was assessed using the Jadad scoring (also called the Oxford quality scoring) system (Table 1).

\section{Results}

A total of 10 randomized controlled trials (RCTs) met our inclusion and exclusion criteria. Three studies included only female subjects, 2 studies included only male subjects, and 5 included both. Five different laser devices were used. Jadad scores (Table 1) ranged from 1 to 5 , with a mean of 4 . Each study is described below and summarized in Table 2.

Leavitt et al. [18] described the use of the HairMax Laser Comb device for treating AGA. The 110 patients were all males and were classified with Norwood-Hamilton MPHL scores (IIa-V). In this randomized, double-blinded, sham device-controlled, multicenter trial, the subjects were treated with either the HairMax Laser Comb or a sham device (2:1 ratio). The patients were asked to use the device 3 times a week for $15 \mathrm{~min}$ on non-concurrent days for the duration of 26 weeks/ 6 months. Although investigators' assessment of overall hair growth showed no significant difference between the groups, treatment with the HairMax Laser Comb had a mean increase in terminal hair density of +19.8 hairs $/ \mathrm{cm}^{2}$ compared to the sham device that had a mean decrease of -7.6 hairs $/ \mathrm{cm}^{2}$ at 26 weeks. These results were statistically significant $(p<$ 0.0001). Further, the HairMax Laser Comb group perceived a greater improvement in hair regrowth at 26 weeks ( $p=0.01)$ and had a more favorable overall assessment according to the subjects' study questionnaire. Other than 4 cases of mild paresthesia and 4 cases of mild urticaria, the treatment with the HairMax Laser Comb was well tolerated with no severe complications reported. This study suggests that the HairMax Laser Comb device was not only perceived to have a greater improvement in hair regrowth but was also effective in increasing terminal hair density in males.

Kim et al. [19] described the use of the Oaze helmettype 3R LLLT device for treating AGA and FPHL. In this randomized, double-blinded, sham device-controlled, multicenter trial, 40 subjects including both female and male patients were initially enrolled and treated with either the Oaze helmet-type 3R LLLT device or a sham device (1:1 ratio) for a duration of $18 \mathrm{~min}$, once a day for 24 
weeks. Male patients were classified with NorwoodHamilton MPHL scores (III-IV), and female patients were classified with Ludwig scores (I-III). Primary and secondary endpoints were assessed for a total of 29 patients (LLLT, $n=15$; sham device, $n=14$ ). Treatment with the Oaze helmet-type 3R LLLT device had a statistically significant mean increase in terminal hair density of $+17.2 \pm 12.1$ hairs $/ \mathrm{cm}^{2}$ compared to the treatment with the sham device that had a mean decrease of $-2.1 \pm 18.3$ hairs $/ \mathrm{cm}^{2}$ at 24 weeks $(p<0.003)$. Furthermore, treatment with the Oaze helmet-type 3R LLLT device had a statistically significant mean increase in hair thickness of $+12.6 \pm 9.4 \mu \mathrm{m}$ compared to the treatment with the sham device that had a mean increase of $+3.9 \pm 7.3 \mu \mathrm{m}$ at 24 weeks $(p<0.01)$. Investigators perceived a significantly greater improvement of hair regrowth in the treatment group versus the control group $(p<0.05)$. Subjects' global assessment of hair growth and subjects' satisfaction were not statically significant. The treatment with the Oaze helmet-type 3R LLLT device resulted in no severe complications. Reported adverse effects in both the treatment and control groups included headache, skin pain, pruritis, erythema, and acne; however, there was no significant difference in incidence of adverse events between the 2 groups. The study was a sham device-controlled trial, suggesting that Oaze helmet-type 3R LLLT device was very effective in increasing terminal hair density and hair thickness in both females and males.

Jimenez et al. [17] described the use of HairMax Laser Comb device for treating AGA and FPHL. A total of 128 males with Norwood-Hamilton Baldness scores IIa-V and 141 female patients with Ludwig-Savin Baldness score I-4, II-1, II-2, or frontal were included in this large randomized, double-blinded, multicenter, sham devicecontrolled study. The subjects were randomly treated with either the HairMax Laser Comb device with 7, 9, or 12 laser beams or a sham device 3 times a week for 26 weeks. The treatment with the HairMax Laser Comb device showed a statistically significant increase in terminal hair density from baseline in all treatment groups compared to the treatment with the sham device at 26 weeks. Further, a higher percentage of subjects in the treatment group perceived overall improvement of hair loss condition, thickness, and fullness of hair elicited via self-assessment compared to the control group. Side effects reported included dry skin (5.1\%), pruritis (2.5\%), scalp tenderness $(1.3 \%)$, irritation (1.3\%), and a warm sensation at the site $(1.3 \%)$. There were no side effects that lead to discontinuation or interruption of the study. This study is the largest published randomized, double-blinded, sham de-

Low-Level Laser Therapy for Male and Female Pattern Hair Loss vice-controlled study to date and suggests evidence for the efficacy and safety of the HairMax Laser Comb device in treating AGA and FPHL, increasing terminal hair density in both male and female patients.

Lanzafame et al. [20] described the use of the iGrow (TOPHAT655 unit) helmet-type device for treating AGA. In this randomized, double-blinded, sham device-controlled trial, 41 men with Norwood-Hamilton Baldness scores IIa-V were treated with either the iGrow (TOPHAT655 unit) device or a sham device (1:1 ratio). The patients were asked to use the device for 25 min every other day for 16 weeks. Treatment with the iGrow (TOPHAT655 unit) LLLT device had a statistically significant mean percent hair count increase of $+39 \%$ compared to the treatment with the sham device at 16 weeks (67.2 \pm 33.4 , LLLT, $n=22 ; 28.4 \pm 46.2$, sham, $n=19 ; p=$ 0.001 ). No severe complications, adverse events, or side effects were reported. Lanzafame et al. [20] suggested iGrow (TOPHAT655 unit) helmet-type device to be a very efficacious and safe tool that increases hair counts form baseline and improves AGA in male patients.

Lanzafame et al. [21] described the use of the iGrow (TOPHAT655 unit) helmet-type device for treating FPHL. In this randomized, double-blinded, sham devicecontrolled trial, 42 female patients with Ludwig-Savin Baldness scores (I-2-II-2) were treated with either the iGrow (TOPHAT655 unit) device or a sham device (1:1 ratio). The patients were asked to use the device for 25 min every other day for 16 weeks. Treatment with the iGrow (TOPHAT655 unit) LLLT device resulted in a statistically significant mean percent hair growth increase of $+48.07 \pm 17.61$ compared to the treatment with the sham device with a mean percent hair growth increase of $+11.05 \pm 48.30$ at 16 weeks $(p<0.001)$. This correlates to a $37 \%$ increase in hair growth in the treatment versus the control group. No severe complications, adverse events, or side effects were associated with the iGrow (TOPHAT655 unit) LLLT device. Similar to their previous study conducted in male patients only, Lanzafame et al. [21] advocated for safety and efficacy of the iGrow (TOPHAT655 unit) helmet-type device to improve hair growth in female patients.

Friedman and Schnoor [22] described the use of the Capillus Handi-Dome Laser device for treating FPHL. In this randomized, double-blinded, sham device-controlled trial, 44 female patients with Ludwig-Savin Baldness scores (I-2-II-2) were treated with either the Capillus Handi-Dome Laser device or a sham device (1:1 ratio). The patients were asked to use the device for 30 min every other day for 17 weeks. Treatment with the Capillus Han- 
di-Dome Laser device resulted in a statistically significant increase in terminal hair counts of $51 \%$ compared to the treatment with the sham device at 17 weeks $(p<0.001)$. The treatment with the Capillus Handi-Dome Laser device resulted in no severe complications and no adverse events or side effects were reported at any time during the study. This study indicated that Capillus Handi-Dome Laser device successfully increased terminal hair counts and improved FPHL in female patients.

Barikbin et al. [23] described the use of the red light laser hat and the red light laser scanner for treating AGA and FPHL. In this randomized, double-blinded, controlled study, a total of 90 female and male patients were divided into 3 equal groups - Group 1: patients received $655 \mathrm{~nm}$ red light via laser hat $(n=30)$; Group 2: patients received $655 \mathrm{~nm}$ red laser plus $808 \mathrm{~nm}$ infrared laser using a laser scanner of hair growth device $(n=30)$; and Group 3: patients received no laser as the control group $(n=30)$. Patients were treated 3 times a week for up to 4 months. A statistically significant increase in the number of terminal hairs was observed in both treatment groups $(p<0.0001)$. The treatment with a laser scanner resulted in higher increase in terminal hair density compared to the treatment with the laser hat (mean increase in terminal hair density 9.61 vs. $9.16 / \mathrm{cm}^{2}$ ). These results were statically significant $(p<0.0001)$. No adverse effects were observed in this study. This study results showed that both red light laser hat and red light laser scanner successfully increased terminal hair density and improved AGA without any significant adverse effects.

Mai-Yi Fan et al. [24] described the use of the iRestore ID-520 helmet-type LLLT device for treating AGA and FPHL. In this randomized, double-blinded, self-comparison, sham device-controlled study, 100 subjects including male patients with Norwood-Hamilton male pattern hair loss scores IIa-V and female patients with LudwigSavin classification scores I4-II-2 were treated with the iRestore ID-520 helmet-type LLLT device on one side of the head, and a sham device on the contralateral side. Half of the subjects were treated with the iRestore ID-520 helmet-type LLLT device on the right side and the sham device on the left side, and the other half of the subjects with the opposite light sources on their respective sides. The patients were asked to use the device for $30 \mathrm{~min} 3$ times a week for 24 weeks. The side treated with the iRestore ID520 helmet-type LLLT device resulted in a significantly greater hair coverage compared to the side treated with the sham device ( 14.2 vs. $11.8 \%)$ at 24 weeks $(p<0.001)$. Furthermore, the side treated with the iRestore ID-520 helmet-type LLLT device resulted in greater improve- ment in hair thickness, hair count, hair coverage, and the investigators' global assessment of hair regrowth compared to the side treated with the sham device at 24 weeks. These results were all statistically significant $(p<0.001$, $p<0.001, p<0.001$, and $p<0.001$, respectively). Subjects global assessment of hair growth and subjects' satisfaction were not statically significant. There were no cases of severe complications, interruption of the study, or discontinued use of the study device. Adverse effects were reported in 29 patients $(29.3 \%)$ and included eczema $(4.0 \%)$, pruritis $(3.0 \%)$, and acne (1.0\%). Most of the aforementioned adverse events resolved within 2 weeks. This study suggests that the iRestore ID-520 helmet-type LLLT device successfully increased hair coverage, hair thickness, and hair count and resulted in a better global assessment of hair regrowth by the investigators without causing any serious side effects.

Esmat et al. [25] described the use of the iGrow LLLT helmet device for treating FPHL. In this randomized, double-blinded, controlled study, 45 female patients with a diagnosis FPHL and Ludwig-Savin Baldness scores I-III were divided into 3 equal groups - Group A: patients were asked to apply topical minoxidil 5\% twice a day for 4 months; Group B: patients used the iGrow LLLT helmet device for 25 min every other day for 4 months; and Group C: patients received both topical minoxidil 5\% twice a day and the iGrow LLLT helmet device for $25 \mathrm{~min}$ every other day for 4 months. A statically significant increase in the number of hair follicles at 4 months was observed in both Group B and Group C. There was no significant difference between the diameters of the largest hair follicle in any of the 3 groups. The treatment with the iGrow LLLT helmet device resulted in no severe complications. Side effects reported include self-limiting irritation (27\% of patients in Group C vs. $40 \%$ of patients in Group A), scalp tenderness (27\% of patients in Group B vs. $40 \%$ of patients in Group C), warm sensation ( $20 \%$ of patients in group B vs. $27 \%$ of patients in group C), and initial increase in hair shedding ( $80 \%$ of patients in group A vs. 60 of patients in group C). These results suggest that the iGrow LLLT helmet device did not increase the diameter of the largest hair follicle but that it effectively increased the number of hair follicles at the end of the treatment period in females suffering from FPHL with a satisfactory side effect profile.

Suchonwanit et al. [26] described the use of the RAMACAP helmet-type LLLT device for treating AGA and FPHL. In this randomized, double-blinded, sham devicecontrolled study, 36 total subjects including both female patients with Ludwig-Savin Baldness score I-III and male 
patients with Norwood-Hamilton Baldness scores III-V were treated with either the RAMACAP helmet-type LLLT device or a sham device (10 men and 10 women in each group). The patients were asked to use the device for 20 min 3 times per week for 24 weeks. Treatment with the RAMACAP helmet-type LLLT device resulted in a statistically significant increase in terminal hair counts of $51 \%$ compared to the treatment with the sham device at 17 weeks $(p<0.001)$, as well as a statistically significant increase in hair diameter at 24 weeks $(p=0.009)$. Both investigators and subjects perceived greater improvement in hair regrowth in the treatment group versus the control group, and the results were statistically significant ( $p=$ 0.0002 and $p=0.0026$, respectively). The treatment with the RAMACAP helmet-type LLLT device resulted in no adverse events, discontinuation, or interruption of the study. Overall, Suchonwanit et al. [26] proposed that the RAMACAP helmet-type LLLT device demonstrated the ability to safely improve AGA and FPHL by increasing terminal hair counts and hair diameter.

\section{Discussions}

The purpose of our systematic review was to evaluate the published controlled clinical trials utilizing LLLT to determine if the data support its use for treatment of pattern hair loss. Overall, the 10 RCTs suggested satisfactory results following laser treatment and most devices had favorable safety profiles. All of the trials found significant increases in terminal hair counts, hair growth, and hair coverage in treatment groups $(p<0.01)$.

All 10 RCTs included in this review had control groups, and the studies were carried out for at least 16 weeks, with the longest treatment period being 26 weeks. Furthermore, all of the studies employed objective measurements of hair loss or regrowth: the Norwood-Hamilton scale for men and the Ludwig-Savin scale for women. Eight out of the 10 studies used sham devices as controls. Of the 2 that did not use sham devices, minoxidil was used for one treatment group [25] and the other, no treatment was given to the control group [23].

The data acquired from each study used different devices, laser parameters, and regimens. Various laser devices were utilized, including the HairMax Laser Comb device, the Oaze helmet-type 3R LLLT device, the iGrow (TOPHAT655 unit) helmet-type device, and several others. Laser wavelengths included $660 \pm 10$ and $655 \mathrm{~nm}$ (Table 2). Though each study developed their treatment standards, one benefit of this review is that laser parameters

Low-Level Laser Therapy for Male and Female Pattern Hair Loss and regimens were reported by all but one of the RCTs, so comparisons can be made. Four of the RCTs $[17,18$, 24, 26] implemented laser treatment 3 times per week for between 15 and 25 min depending on the study. Another 4 studies [20-22, 25] required treatment every other day for 25 or $30 \mathrm{~min}$. One study [19] chose to have participants treated every day. Of note, subjects' assessment of satisfaction was not favorable for this study, perhaps due to daily treatment requirements.

Only one study reviewed [25] and compared commonly prescribed pharmacological treatments for AGA with LLLT. In this case, minoxidil 5\% topical solution was used. This study included 3 treatment groups: one in which patients applied minoxidil twice a day for 16 weeks, one in which patients used the iGrow LLLT helmet device every other day, and a third group that used both minoxidil and the laser. This study suggested that both the group that used laser alone and the group that used laser in combination with minoxidil showed significant increases in the number of hair follicles at the end of treatment $(p<0.05)$. Moreover, the results demonstrated that LLLT alone did result in slightly better outcome than minoxidil alone, while combination therapy was found to be superior to either treatments alone, proposing an important role of LLLT in positive outcomes.

In order to objectively measure the efficacy of LLLT, researchers used standardized forms of imaging and counting techniques to assess hair density before and after treatment. In most cases, a location of interest was identified on the scalp and hairs were trimmed in a circular area ranging from approximately 1 to $3 \mathrm{~cm}^{2}$. A pinpoint tattoo was then placed at the center of the clipped area for use as a guide in order to clip and image the same area at subsequent visits. In 4 of the studies [19,24-26], a phototrichogram was used for measurement, either a Folliscope ${ }^{\circledR}$ (Lead M, Seoul, South Korea) or an IBS-01 Pro Beauty Scope (Kowa Optics Corp., New Taipei City, Taiwan). Three of the studies [20-22] used a custom-built camera apparatus consisting of a Canon Rebel T3i camera system (Canon, Melville, NY, USA) equipped with a Tamron macro lens (Tamron, Commack, NY, USA). In general, hair counts were performed by a single blinded observer and in most cases, images sent for independent count via a computer-assisted software.

In evaluating LLLT for AGA, we must consider not only the efficacy of laser devices, but the safety of their use as well. Most subjects in the studies reported only mild side effects, commonly including pruritis $[17,19,24]$, acne $[19,24]$, and scalp tenderness $[17,25]$. No studies reported any severe adverse events that lead to disruption 
or discontinuation of treatment, and most of the aforementioned side effects resolved within 2 weeks. Another important aspect to consider in the use of LLLT is the satisfaction of patients following treatment. Five of the studies included assessment of patient satisfaction with treatment. In most cases, treatment groups were found to have a more favorable overall assessment when compared to control groups. Moreover, LLLT devices appear to be a safe and efficacious form of treatment for AGA. They are of relatively low cost, are easy to use, and are therefore likely to result in high levels of compliance. Although all 10 studies suggest LLLT devices to also be very effective, there are a couple of significant topics we would like to discuss.

First, it is important to note that some of the studies included in this review have a clear relation with an industry; the relationship includes either sponsoring the study or involvement in evaluating the results of the study. It is then possible that such relation can affect the validity of the study designs, results, and/or conclusions. Second, the automated digital imaging analysis or the Trichoscan may be an easy method to evaluate hair growth but has been shown as imperfect and error-prone [27]. That said, although Trichoscan evaluations suggest promising results in terms of the numbers (i.e., increase in hair density and hair thickness), the articles included have shown little clinical improvement overall. Given the increase in terminal hair counts in all studies, perhaps a longer duration of treatment could lead to clinical improvement. That said, although LLLT may be an alternative safe and effective tool for treating pattern hair loss, more studies with additional focus on clinical improvements, comparison of diverse laser settings, and treatment regimens will be vital for dermatologists to develop a more standardized treatment regimen that yields maximum efficacy for patients with pattern hair loss. Furthermore, dermatologists should look out for any potential connections with industries when evaluating the literature and making decisions based on a particular clinical trial when it comes to both efficacy and safety.

\section{Conflict of Interest Statement}

The authors have no conflicts of interest to declare.

\section{Funding Sources}

The authors did not receive any funding.

\section{Author Contributions}

Every author listed meets the qualifications for authorship and has had the opportunity to read and comment upon the submitted manuscript.

\section{References}

1 Otberg N, Finner AM, Shapiro J. Androgenetic alopecia. Endocrinol Metab Clin North Am. 2007 Jun;36(2):379-98.

2 Gan DC, Sinclair RD. Prevalence of male and female pattern hair loss in Maryborough. J Investig Dermatol Symp Proc. 2005 Dec;10(3): 184-9.

3 Wells PA, Willmoth T, Russell RJ. Does fortune favour the bald? Psychological correlates of hair loss in males. Br J Psychol. 1995 Aug; 86(Pt 3):337-44.

4 Cash TF. The psychosocial consequences of androgenetic alopecia: a review of the research literature. Br J Dermatol. 1999 Sep; 141(3):398-405.

5 Williamson D, Gonzalez M, Finlay AY. The effect of hair loss on quality of life. J Eur Acad Dermatol Venereol. 2001 Mar;15(2): 137-9.

6 Cash TF. The psychological effects of androgenetic alopecia in men. J Am Acad Dermatol. 1992 Jun;26(6):926-31.

7 Cash TF, Price VH, Savin RC. Psychological effects of androgenetic alopecia on women: comparisons with balding men and with fe- male control subjects. J Am Acad Dermatol. 1993 Oct;29(4):568-75.

8 Jahoda CA, Horne KA, Oliver RF. Induction of hair growth by implantation of cultured dermal papilla cells. Nature. 1984 Oct 11-17; 311(5986):560-2.

9 Avci P, Gupta GK, Clark J, Wikonkal N, Hamblin MR. Low-level laser (light) therapy (LLLT) for treatment of hair loss. Lasers Surg Med. 2014 Feb;46(2):144-51.

10 Rogers NE, Avram MR. Medical treatments for male and female pattern hair loss. J Am Acad Dermatol. 2008 Oct;59(4):547-8.

11 Xu D, Wang L, Dai W, Lu L. A requirement for $\mathrm{K}+$-channel activity in growth factor-mediated extracellular signal-regulated kinase activation in human myeloblastic leukemia ML-1 cells. Blood. 1999 Jul 1;94(1):139-45.

12 Shorter K, Farjo NP, Picksley SM, Randall VA. Human hair follicles contain two forms of ATP-sensitive potassium channels, only one of which is sensitive to minoxidil. FASEB J. 2008 Jun;22(6):1725-36.

13 Adil A, Godwin M. The effectiveness of treatments for androgenetic alopecia: a systematic review and meta-analysis. J Am Acad Dermatol. 2017 Jul;77(1):136-141.e5.

14 Dodd EM, Winter MA, Hordinsky MK, Sadick NS, Farah RS. Photobiomodulation therapy for androgenetic alopecia: a clinician's guide to home-use devices cleared by the Federal Drug Administration. J Cosmet Laser Ther. 2018 Jun;20(3):159-67.

15 Wikramanayake TC, Rodriguez R, Choudhary S, Mauro LM, Nouri K, Schachner LA, et al. Effects of the Lexington LaserComb on hair regrowth in the $\mathrm{C} 3 \mathrm{H} / \mathrm{HeJ}$ mouse model of alopecia areata. Lasers Med Sci. 2012 Mar; 27(2):431-6.

16 Kalia S, Lui H. Utilizing electromagnetic radiation for hair growth: a critical review of phototrichogenesis. Dermatol Clin. 2013 Jan; 31(1):193-200.

17 Jimenez JJ, Wikramanayake TC, Bergfeld W, Hordinsky M, Hickman JG, Hamblin MR, et al. Efficacy and safety of a low-level laser device in the treatment of male and female pattern hair loss: a multicenter, randomized, sham device-controlled, double-blind study. Am J Clin Dermatol. 2014 Apr;15(2):115-27. 
18 Leavitt M, Charles G, Heyman E, Michaels D. HairMax LaserComb laser phototherapy device in the treatment of male androgenetic alopecia: a randomized, double-blind, sham device-controlled, multicentre trial. Clin Drug Investig. 2009;29(5):283-92.

19 Kim H, Choi JW, Kim JY, Shin JW, Lee SJ, Huh CH. Low-level light therapy for androgenetic alopecia: a 24-week, randomized, double-blind, sham device-controlled multicenter trial. Dermatol Surg. 2013 Aug;39(8): 1177-83.

20 Lanzafame RJ, Blanche RR, Bodian AB, Chiacchierini RP, Fernandez-Obregon A, Kazmirek ER. The growth of human scalp hair mediated by visible red light laser and LED sources in males. Lasers Surg Med. 2013 Oct; 45(8):487-95.
21 Lanzafame RJ, Blanche RR, Chiacchierini RP, Kazmirek ER, Sklar JA. The growth of human scalp hair in females using visible red light laser and LED sources. Lasers Surg Med. 2014 Oct;46(8):601-7.

22 Friedman S, Schnoor P. Novel approach to treating androgenetic alopecia in females With photobiomodulation (low-level laser therapy). Dermatol Surg. 2017 Jun;43(6): 856-67.

23 Barikbin B, Khodamrdi Z, Kholoosi L, Akhgri MR, Haj Abbasi M, Hajabbasi M, et al. Comparison of the effects of $665 \mathrm{~nm}$ low level diode Laser Hat versus and a combination of $665 \mathrm{~nm}$ and $808 \mathrm{~nm}$ low level diode Laser Scanner of hair growth in androgenic alopecia. J Cosmet Laser Ther. 2017 [Online ahead of print].

24 Mai-Yi Fan S, Cheng YP, Lee MY, Lin SJ, Chiu HY. Efficacy and safety of a low-level light therapy for androgenetic alopecia: a 24-week, randomized, double-blind, self-comparison, sham device-controlled trial. Dermatol Surg. 2018 Nov;44(11):1411-20.
25 Esmat SM, Hegazy RA, Gawdat HI, Abdel Hay RM, Allam RS, El Naggar R, et al. Low level light-minoxidil 5\% combination versus either therapeutic modality alone in management of female patterned hair loss: a randomized controlled study. Lasers Surg Med. 2017 Nov;49(9):835-43.

26 Suchonwanit P, Chalermroj N, Khunkhet S. Low-level laser therapy for the treatment of androgenetic alopecia in Thai men and women: a 24-week, randomized, double-blind, sham device-controlled trial. Lasers Med Sci. 2019 Aug;34(6):1107-14.

27 Saraogi PP, Dhurat RS. Automated Digital Image Analysis (TrichoScan ${ }^{\circledR}$ ) for human hair growth analysis: ease versus errors. Int J Trichology. 2010 Jan;2(1):5-13. 